\title{
EMG ONSET DETECTION - A HIDDEN FACTOR
}

\author{
Mateusz Magda ${ }^{1}$
}

\begin{abstract}
Electromyographic (EMG) examination has gained popularity and was deployed in both practical and research fields. This technology enables one to observe the voltage generated by a neuromuscular system and to interpret body movements. Over time, it has been proved highly difficult to precisely determine the beginning of a muscle contraction. A relatively low signal to noise ratio, which is even smaller in case of a surface electromyography (sEMG), complicates this task, and more advanced onset detection algorithms were proposed. Despite the existence of statistically advanced algorithms, proposed in recent years, in many cases a manual onset detection performed by an EMG specialist remains in use, mainly due to lack of reliable solutions. This paper presents a hidden factor, not found in the literature until now, which directly relates subsequent voltage values with muscle activity. Observation of this parameter helps detect muscle onset precisely. Combined with other statistical factors, it can shed light on a totally new, enhanced branch of muscle onset estimators. This study conducts a numerical comparison to follow the hypothesis that the examined factor is correlated with a muscle contraction state.
\end{abstract}

UDC Classification: 612 DOI: http://dx.doi.org/10.12955/cbup.v4.867

Keywords: EMG, Muscle, Onset, Detection, Factor.

\section{Introduction}

The electromyographic (EMG) signal is an electrical current that can be measured directly inside of a muscle tissue after piercing the skin layer. To facilitate wider use of this technology among different application areas there appeared a method of measuring this signal on the skin surface which is called surface electromyography (sEMG). However, processing the EMG signal captured using this technology is a difficult task as the skin layer is rather electrically resistant, and the task of extracting information from this signal is challenging. A major and essential task would be creating an automatic onset detection method that identifies the onset of a muscle activity as precisely as possible. Even though some statistically advanced algorithms exist in the literature, the manual onset detection in many areas, such as medicine or rehabilitation, have not been replaced. This is because of a lack of reliability. The vast part of existing solutions is tested on mathematical models of an EMG signal, and have not been tested on a sufficient amount of sEMG data. Thus, when a specialist deploys such a solution, they often find the outcome inadequate.

This article presents a "hidden factor" that will help in processing sEMG signals efficiently and build a robust muscle onset estimator. The factor is based on a dependency between subsequent samples' voltage values.

\section{Literature Review}

The first attempt of solving the onset estimation problem was presented by Cavanagh and Komi (1979). This involved a simple threshold based algorithm that compared electrical current values with a previously set threshold and after exceeding the particular value it reported that the muscle has been contracted. This solution was vulnerable because a single voltage peak would destroy the entire concept. However, this approach opened a series of threshold-based algorithms that are now mainstream in this field. The next milestone in this field was from Staude, Flachenecker, Daumer, \& Wolf (2001), who compared and summarized all major existing methods, and presented a new solution, approximated generalized likelihood-ratio detectors step (AGLRstep), as a state of the art algorithm. The same year and the same author tested a whitening filter and its influence on the EMG signal (Staude, 2001). The results of this study were positive. However, it was generally recommended not to apply strong filters in the EMG signal because they would unacceptably deform and shift the onset. A critical phenomenon was described by Mezzarane and Kohn (2009), who noticed EMG "cross-talks", which are EMG signals from adjacent muscles cooperating with the observed muscle in some particular activities. That interaction hinders the entire EMG signal processing and it was crucial

\footnotetext{
${ }^{1}$ Mateusz Magda, Computer Science \& Management, Wroclaw University of Science and Technology, Wroclaw, Poland, mateusz.magda@pwr.edu.pl
} 
to know whether observations in the electromyogram were specifically a muscle activity or a signal noise.

Signals originating from surrounding muscles are generally lower in voltage, can appear in a moment in which we do not expect any electrical activity and are considerably different in characteristics to the signal noise. Solnik, DeVita, Rider, Long, \& Hortobágyi (2008) presented a way of conditioning the sEMG signal with a Teager-Kaiser Energy Operator, which is a type of signal filter that returns magnified values of electrical current in place of high values and diminishes the signal in the lower parts.

A formula for calculating a current frame is expressed as follows:

$$
x(n)=x(n)^{\wedge} 2-x(n-1) * x(n+1)
$$

where $\mathrm{x}(\mathrm{n})$ is the current voltage value

This gives a solution for a filtered signal that is generally easier to identify the onset, though the whole graph becomes distorted and many signal features, including the one presented in this paper, are lost. The next solution worth mentioning is a two-stage onset estimator as presented by Drapala, Brzostowski, Szpala, and Rutkowska-Kucharska (2012). It consists of two subsequent phases. The first phase was called the initial detection and the second - specific detection. The idea of dividing the onset estimation into two separate blocks seemed sound and well-reasoned because of the need for delivering a robust solution.

Rodriguez-Falces, Navallas, and Malanda (2012) in their chapter "EMG Modeling" described several approaches for presenting the EMG signal. Two mathematical models of an EMG signal are of interest to this study. The first, presented by Farina, Mesin, Martina, and Merletti (2004) is a cylindrical EMG model with an enhanced version of this model proposed soon after by Lowery, Stoykov, Dewald, and Kuiken (2004). This model was a 3-dimensional type, based on the magnetic resonance imaging (MRI) technology and could consider the influence of blood flow within the human arm in the EMG signal. Depending on the application one could consider the importance of various criteria. In some cases, implementing a Pareto optimality may allow "uniting efficiency and justice" (He, 2011). Tartakovsky, Nikiforov, and Basseville (2014) wrote about sequential analysis and changepoint detection, proposing several methods that might be deployed in the area of EMG onset detection, that might be divided into two groups. The first group of presented solutions was the probability-based Bayesian detector and the second, the statistically based algorithms similar to the AGLRstep mentioned above.

Among the sources mentioned, there were many different techniques that could directly solve the problem of muscle onset detection. None, however, are accurate enough to replace the manual onset detection that takes place in many applications. Where both high accuracy and high reliability are required, existing solutions tend to fail. High accuracy is achieved at the expense of robustness in particular solutions. The majority of existing onset estimators deploy moving window-frame mechanisms that can provide one with reliable solutions. In this respect, instead of measuring a single value of a current voltage one can measure some type of a statistical factor on a wider range of frames. An example is measuring the mean value or variance of 150 subsequent frames and reporting the onset whenever it has exceeded particular threshold. This avoids identifying an activity prematurely and confusing a single frame of relatively high current value with a signal onset. A high value of particular frame or small group of frames can arise from signal noise or EMG cross-talk from another muscle, as Mezzarane and Kohn (2009) has proven. This study will measure a statistical factor on a range of 150 voltage samples of a muscle activity to reliably identify the exact frame to be marked as the precise beginning.

\section{A Hidden Factor}

Widening a window frame, one can increase the chance of indicating the onset in the correct region, but, at the same time, decrease the precision of a result. A "hidden factor" presented in this paper, along with other concepts described in the discussion, may help achieve higher accuracy with sound level of robustness. 
The hidden factor, which involves multiplication of three subsequent samples, shows where a muscle has begun its activity. The hidden factor was captured during a long-term observation of sEMG signals and is based on the fact the activity state of a muscle that can be observed with larger differences between subsequent samples of an electrical current, measured on the skin surface. Comparing this with the resting state where these measurements are much closer in value we can deduct about the muscle state. The difference is magnified when values lower than one are multiplied. Multiplying by a number between " 0 " and " 1 " decreases the total value. To illustrate this outcome, consider an example of two operations, powering the value of $0.2 \mathrm{mV}$ and the number of $0.02 \mathrm{mV}$, where the result of the first operation will be $0.04 \mathrm{mV}$, while that of the second will be 100 times lower and equal to 0.0004 $\mathrm{mV}$. This outcome facilitates the onset detection. The assumption has been made that the muscle activity does not take place in the first 75 nor in the last 75 milliseconds of signal. Placing a pseudocode of an algorithm is considered a clear way of presenting an idea. Thus, the solution proposed in this paper is described in such a way below.

Calculating a hidden value involves the following pseudocode:

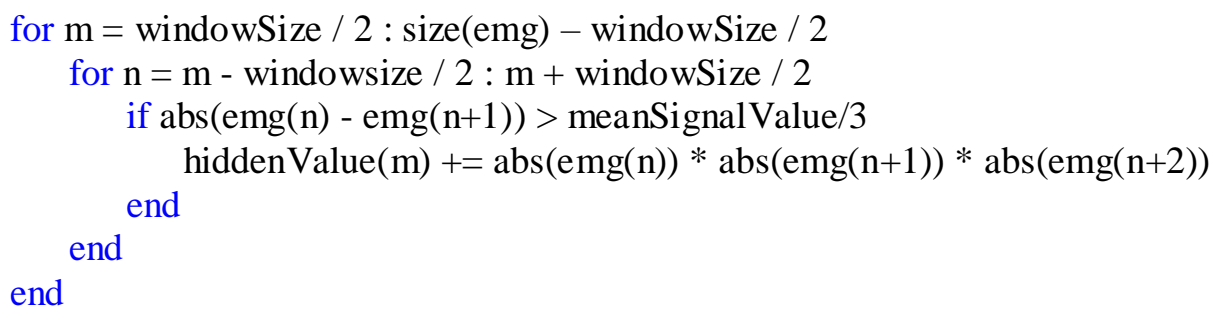

Selecting the onset moment:

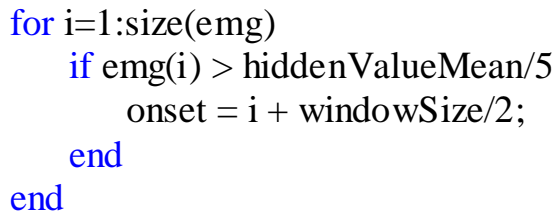

Where:

emg is the examined emg signal

windowSize $=150$

It can seem an enormous drawback to move an obscure window frame in the algorithm to a place where one needs for it to mark the exact onset. It is problematic because a single sample in this solution represents a wider window frame on which the particular statistical value has been measured. It is unclear whether one should mark the beginning, the middle, or the end of a frame as an onset. Since changes in the signal amend the statistical factors rapidly, the option is to mark the end of a frame as the beginning of a change in the signal. Figure 1 illustrates the situation of a moving frame, passing progressively from the beginning of a signal with the position where change is detected shaded in green. It is logical to mark the onset at the end of a frame because this is where the change is signaled in the electrical current.

\section{Data and Methodology}

Data were collected at the University of Physical Education in Wroclaw. Part of the data were precisely described by Szpala, Rutkowska-Kucharska, and Drapala (2014) in an article describing electromechanical delay (EMD). The dataset consisted of 2142 signals from 357 trials, recorded over 30 examinations during two days. The sEMG signals were recorded on the skin surface using six pairs of electrodes and one reference electrode, placed in the electrically passive location.

A count of 240 signals recorded in muscle cells of the erector spinae, were selected for examination in this study. These had been shown to be problematic. All sEMG signals had their onset marked by a specialist before research commenced. All signals were processed by the same algorithm. Indicated onsets were compared to manually marked ones, the differences were calculated and final results prepared. 


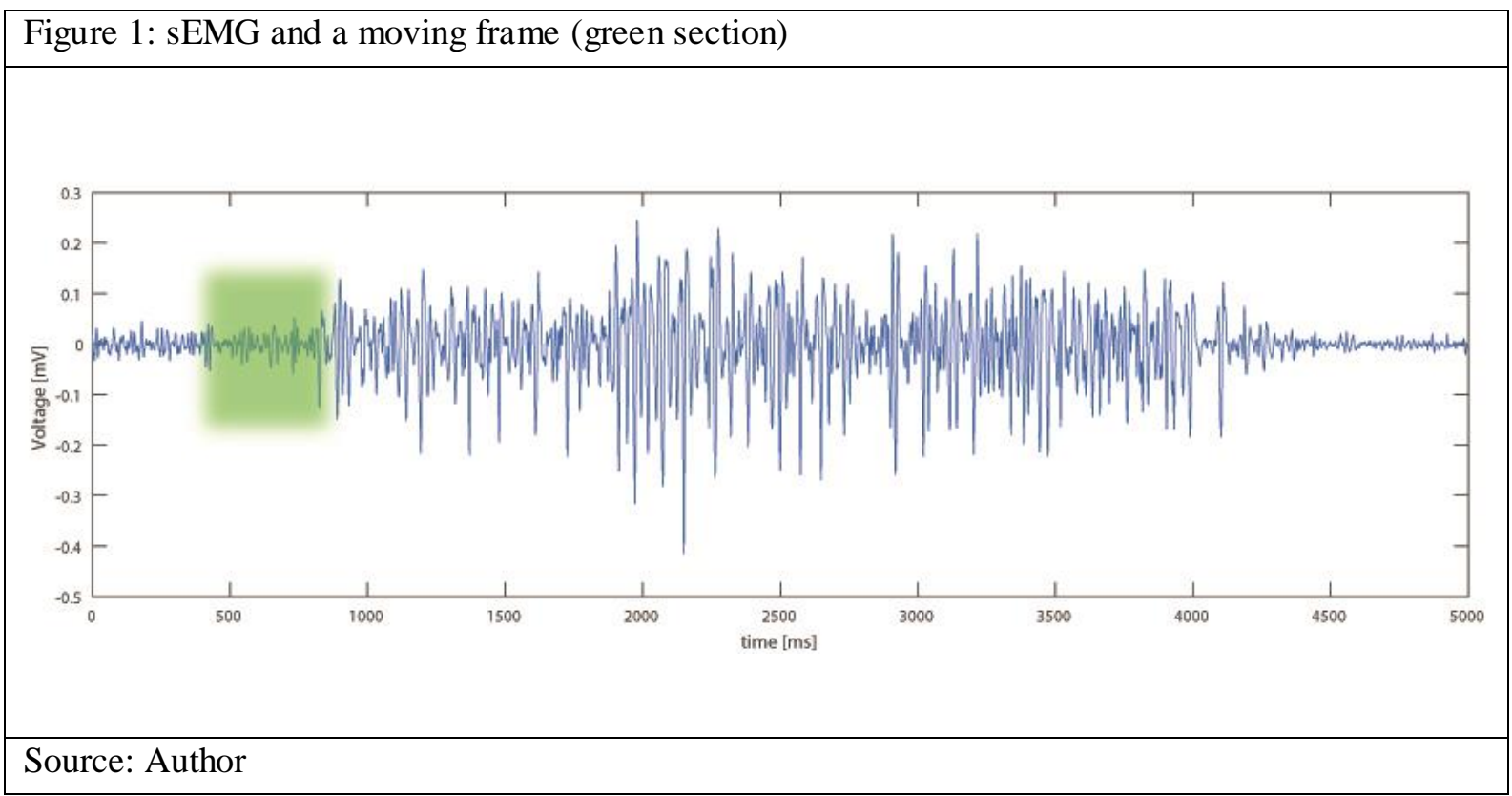

\section{Results and Discussion}

Results of testing the hidden factor as an onset estimator showed a single factor designed as a subsidiary element had a satisfactory level of accuracy. Figure 2 shows the values of sEMG signals (on the left) and their corresponding hidden factor on the right. Comparing the two columns a correlation seems evident. The table 1 presents the actual results.

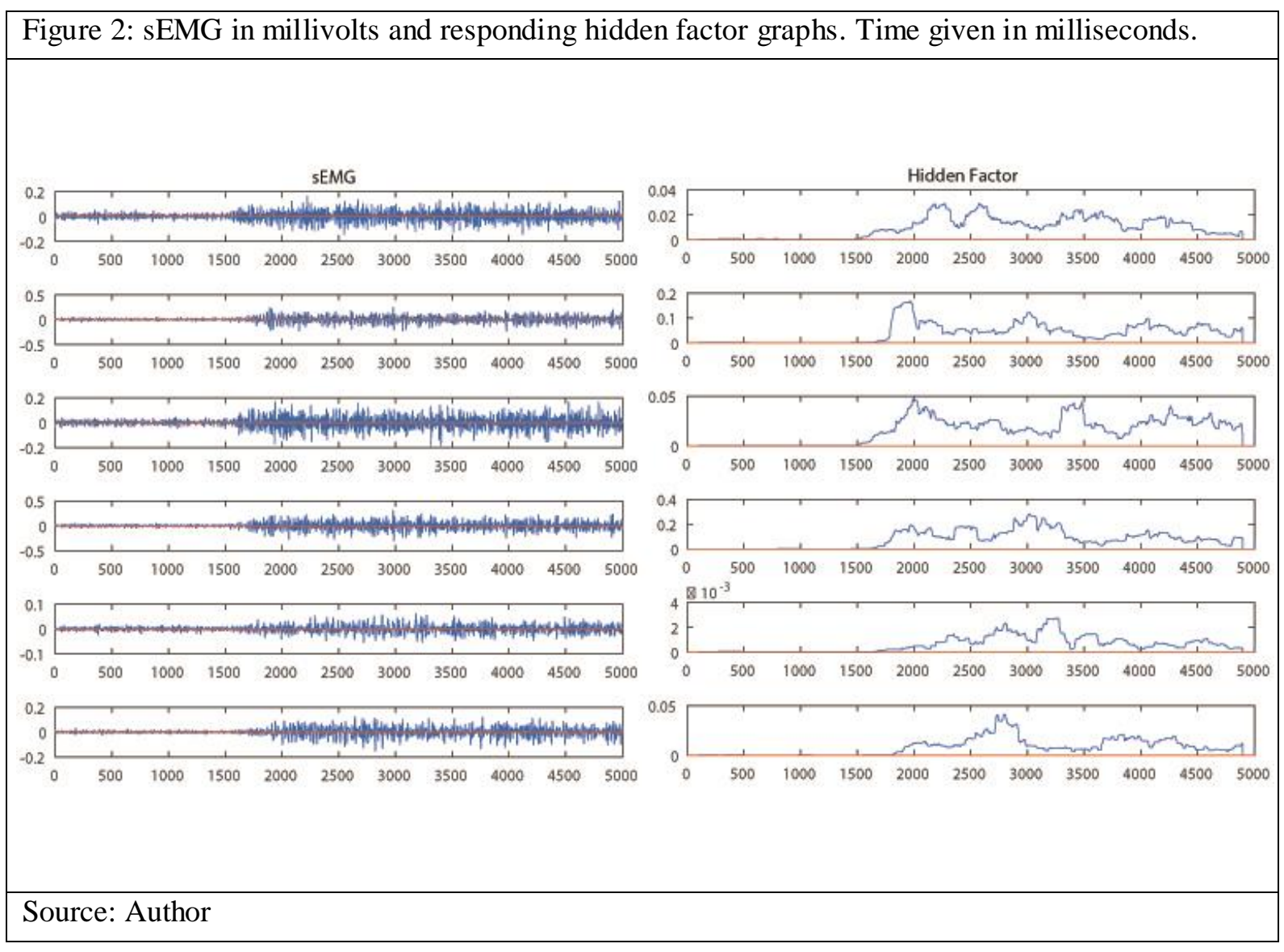

Table 1 shows that over half the estimations were pointed closer than $100 \mathrm{~ms}$ from the actual onset. Only $7 \%$ of the total estimations were pointed further than $400 \mathrm{~ms}$ from that point. The solution has potential as a subsidiary criterion in a more advanced algorithm. The overall mean error rate for this algorithm was $7.86 \mathrm{~ms}$. 


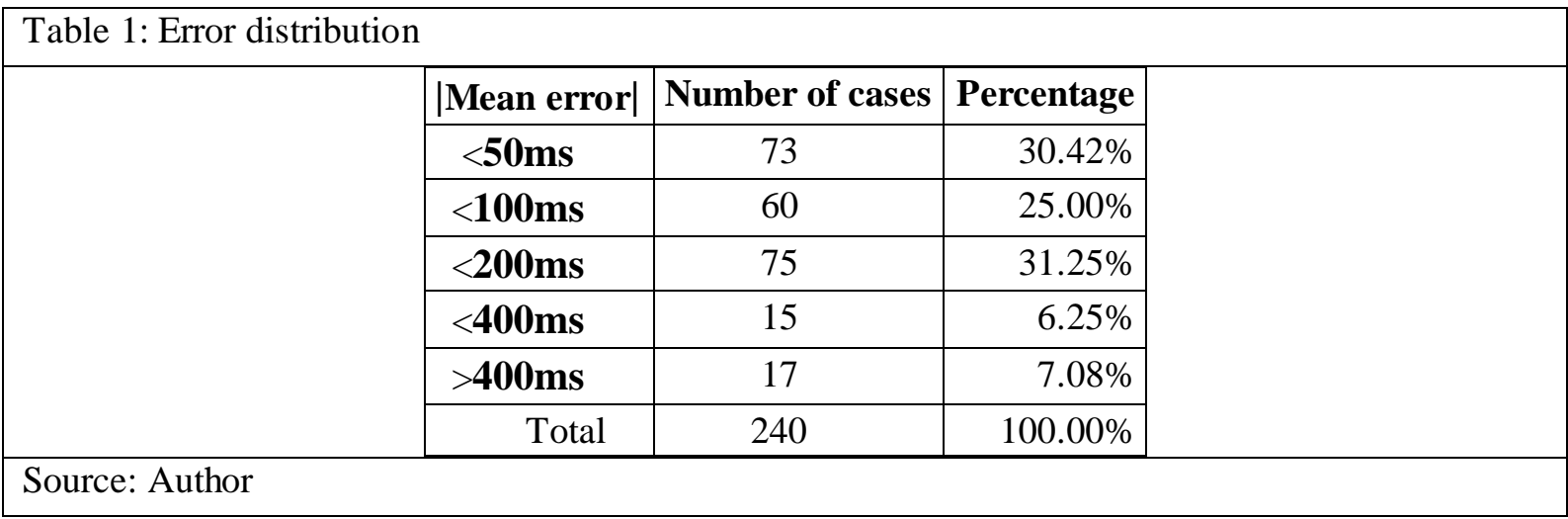

The histogram (Figure 3) shows the distribution of all errors produced by the algorithm. It is noted that no automatic parameter fitting solution was used and thus, the results might be greatly improved by simply shifting the onset. The outliers are shown in the left part of the chart. These were most probably the EMG signals violated by cross-talks from other muscles and such cases need more specific treatment. However, the outliers were included to show that some problems still exist. The histogram shows that, in general, the algorithm identified the onset prematurely. We conclude that by improving the parameter fitting process, results would be enhanced and reflected in the histogram.

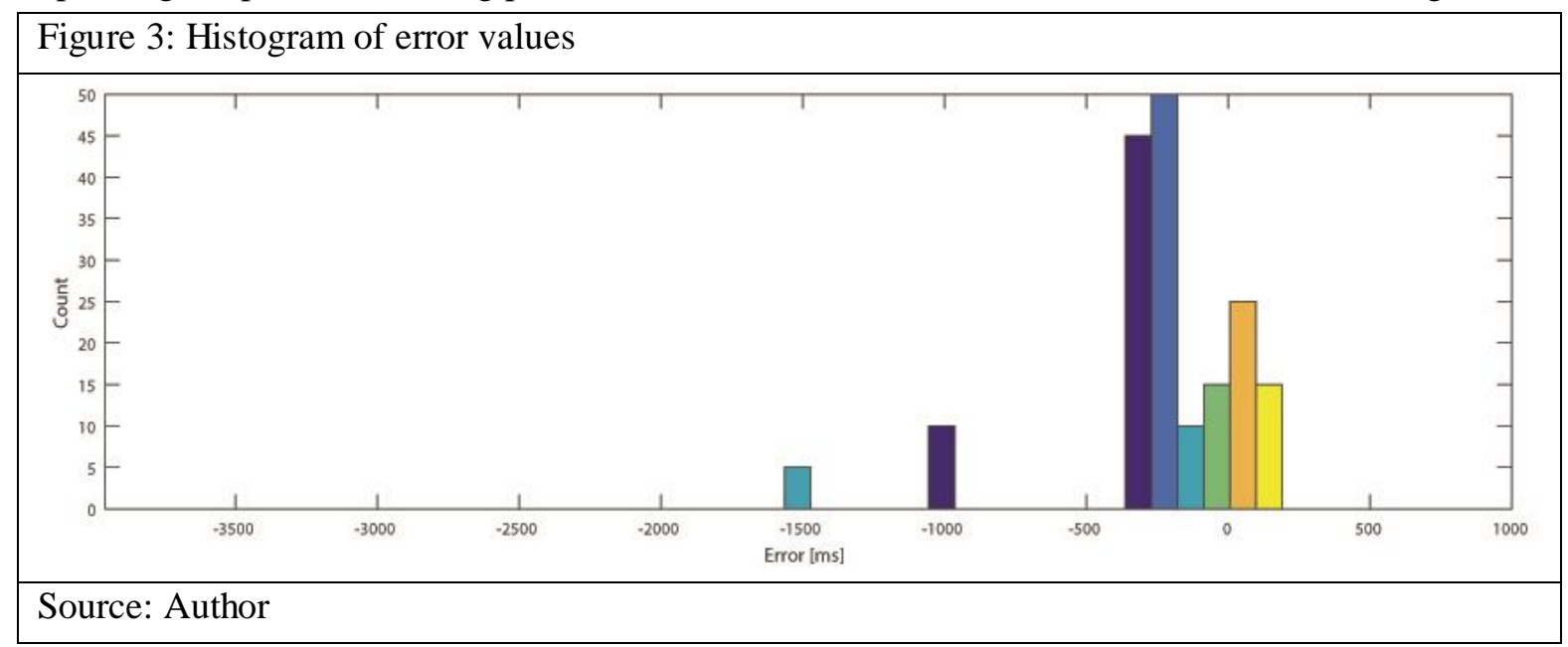

\section{Conclusion}

The results clearly show that multiplying differences between subsequent samples can assist EMG onset detection. Over 55\% of all signals were assessed with accuracy higher than $100 \mathrm{~ms}$. This process could be used to replace the signal filter because it does not shift the actual moment of muscle activity as in case of other solutions. Outliers visible on the histogram (Figure 3), though low in number (7.08\% of total results), indicate problems still exist. Combining the hidden factor with another criterion of EMG onset detection could produce a more robust solution. Thus, the presented phenomenon should be considered as part of a larger solution rather than a standalone algorithm for onset detection.

Furthermore, not only two or three subsequent values but more samples might be considered useful and help distinguish states of an examined muscle. Nevertheless, increasing the number of samples will reduce accuracy because identifying the sample of the actual beginning from a larger set is more problematic. In addition, the presented hidden factor might be useful in bio realistic mathematical models as an additional factor, increasing reliability. To increase robustness of an estimator solution, a mixture of detection by mathematical modeling and statistical analysis could be considered. In the application areas that allow for extended processing, the initial indication could focus on a moving frames method, separating the region of an onset from the whole signal. In the second phase, an isolated onset zone could be used to divide signal into two sections, one when the muscle is resting and the other when it is activated. This could mean applying the classification methods known within the artificial intelligence realm. 


\section{References}

Cavanagh, P. R., \& Komi, P. V. (1979). Electromechanical delay in human skeletal muscle under concentric and eccentric contractions. European Journal of Applied Physiology and Occupational Physiology, 42(3), 159-163.

Drapała, J., Brzostowski, K., Szpala, A., \& Rutkowska-Kucharska, A. (2012). Two stage EMG onset detection method. Archives of Control Sciences, 22(4), 427-440.

Farina, D., Mesin, L., Martina, S., \& Merletti, R. (2004). A surface EMG generation model with multilayer cylindrical description of the volume conductor. IEEE Transactions on Bio-Medical Engineering, 51(3), 415-426. http://DOI.org/10.1109/TBME.2003.820998

He, Z. (2011). Pareto-optimality is a criterion uniting efficiency and justice. In 2011 IEEE 18Th International Conference on Industrial Engineering and Engineering Management (IE EM) (Vol. Part 1, pp. 406-408). http://DOI.org/10.1109/ICIEEM.2011.6035186

Lowery, M. M., Stoykov, N. S., Dewald, J. P. A., \& Kuiken, T. A. (2004). Volume conduction in an anatomically based surface EMG model. IEEE Transactions on Biomedical Engineering, 51(12), 2138-2147. http://DOI.org/10.1109/TBME.2004.836494

Mezzarane, R. A., \& Kohn, A. F. (2009). A method to estimate EMG crosstalk between two muscles based on the silent period following an H-reflex. Medical Engineering \& Physics, 31(10), 1331-1336. http://DOI.org/10.1016/j.medengphy.2009.09.005

Rodriguez-Falces, J., Navallas, J., \& Malanda, A. (2012). Applications, Challenges, and Advancements in Electromyography Signal Processing. IGI Global. http://dx.DOI.org/10.5772/50304

Solnik, S., DeVita, P., Rider, P., Long, B., \& Hortobágyi, T. (2008). Teager-Kaiser Operator improves the accuracy of EMG onset detection independent of signal-to-noise ratio. Acta of Bioengineering and Biomechanics / Wrocław University of Technology, 10(2), 65-68.

Staude, G., Flachenecker, C., Daumer, M., \& Wolf, W. (2001). Onset Detection in Surface Electromyographic Signals: A Systematic Comparison of Methods. EURASIP J. Appl. Signal Process., 2001(1), 67-81. http://DOI.org/10.1155/S1110865701000191

Staude, G. H. (2001). Precise onset detection of human motor responses using a whitening filter and the log-likelihood-ratio test. IEEE Transactions on Biomedical Engineering, 48(11), 1292-1305. http://DOI.org/10.1109/10.959325

Szpala, A., Rutkowska-Kucharska, A., \& Drapala, J. (2014). Electromechanical delay of abdominal muscles is modified by low back pain prevention exercise. Acta of Bioengineering and Biomechanics / Wrocław University of Technology, 16(3), 95-102.

Tartakovsky, A., Nikiforov, I., \& Basseville, M. (2014). Sequential Analysis: Hypothesis Testing and Changepoint Detection. CRC Press. 